\title{
On the use of the sunspot number for the estimation of past solar and upper atmosphere conditions from historical and modern auroral observations
}

\author{
Comment on Schröder, W., Shefov, N. N., and Treder, H.-J.: Estimation of past solar and upper \\ atmosphere conditions from historical and modern auroral observations, Ann. Geophys., 22, \\ 2273-2276, 2004.
}

\author{
J. M. Vaquero \\ Departamento de Física, Escuela Politécnica, Universidad de Extremadura, 10071 Cáceres, Spain
}

Received: 2 November 2005 - Accepted: 14 February 2005 - Published: 28 July 2005

\begin{abstract}
In this short contribution the use of different sunspot numbers for the estimation of past solar and upper atmosphere conditions from historical and modern auroral observations realised by Schröder et al. (2004) is analysed. Moreover, some comments are made on the relationships between mean annual visual observations of the auroras at middle latitudes of Europe and the mean annual sunspot number during 1780-1829.
\end{abstract}

Keywords. Atmospheric composition and structure (Airglow and aurora) - Magnetospheric physics (Auroral phenomena, solar wind-magnetosphere interactions) - History of geophysics (Solar-planetary relationship)

\section{Introduction}

Schröder et al. (2004) realised a meritorious attempt to determine quantitative information on solar activity by comparing the data of visual auroral observations with the modern parameter of their luminescence. Using the mean annual number of visual observations of the auroras $N$ at middle latitudes of Europe and mean annual Wolf number $R_{Z}$ during 17801829, they obtained the approximate relationships (Schröder et al. (2004) used the symbol $W$ for the Wolf number and symbols $N$ and $n$ for the observations of auroras in the first and second equation, respectively)

$N=(21 \pm 1) \cdot \frac{R_{Z}}{100} \cdot\left(1+\frac{R_{Z}}{100}\right)$

and

$R_{Z}=(23 \pm 1) \sqrt{N}-50$.

Correspondence to: J. M. Vaquero

(jvaquero@unex.es)
As typically low-latitude auroras have very often a red colour and are caused by the 630-nm emission of atomic oxygen, they used the empirical relationship between the intensity $I_{630}$, the solar radioflux $F_{10.7}$, the index of geomagnetic disturbance $D_{s t}$ and the geomagnetic latitude obtained by Trustte (1968a, 1968b, 1969). Moreover, they used the relationship obtained by Vitinsky et al. (1986) between the solar radioflux and the Wolf number. Thus, they can make estimations of brightness of expected auroras in the 630-nm emission.

\section{The use of different sunspot numbers}

The International Sunspot Numbers (Wolf or Zürich sunspot numbers) have long served as the primary time series defining solar activity since the year 1700 . This time series was derived by $\mathrm{R}$. Wolf in the 19th century and has been maintained by his successors (Waldmeier, 1947; McKinnon, 1987). Wolf defined the sunspot number, $R_{Z}$, as

$R_{Z}=k(10 g+s)$,

where $g$ is the number of sunspot groups, $s$ is the number of individual sunspots, and $k$ is a correction factor for each observer. The daily $R_{Z}$ value is calculated by using only the input from one observer selected as the primary observer for the given period. If the primary observer could not make an observation, then secondary, tertiary, etc., observers are used to complete the maximum possible number of days. For years up to 1817 , the number of missing days was so great that Wolf only tabulated monthly means. There are no observations for many months from 1749 to 1818 , and for a few months after 1818. Wolf filled in these months by interpolation and using magnetic needle observations. Thus, the numbers $R_{Z}$ are a mixture of direct sunspot observations and calculated values. 
Table 1. The mean annual visual observations of the auroras $N$ at the middle latitudes of Europe provided by Schröder et al. (2004) and mean annual levels of the solar activity $\left(R_{Z}\right.$, Wolf sunspot number; $R_{G}$, Group sunspot number) during 1780-1829.

\begin{tabular}{|c|c|c|c|}
\hline Year & $N$ & $R_{Z}$ & $R_{G}$ \\
\hline 1780 & 16 & 85 & 55 \\
\hline 1781 & 33 & 68 & 71.1 \\
\hline 1782 & 34 & 38 & 32.9 \\
\hline 1783 & 33 & 23 & 21.1 \\
\hline 1784 & 10 & 10 & 4.8 \\
\hline 1785 & 15 & 24 & 16.0 \\
\hline 1786 & 83 & 83 & 63.3 \\
\hline 1787 & 76 & 132 & 89.2 \\
\hline 1788 & 56 & 131 & 82.5 \\
\hline 1789 & 56 & 118 & 79.7 \\
\hline 1790 & 39 & 90 & 65.1 \\
\hline 1791 & 14 & 67 & 43.2 \\
\hline 1792 & 10 & 60 & 42.0 \\
\hline 1793 & 1 & 47 & 41.0 \\
\hline 1794 & 4 & 41 & 30.2 \\
\hline 1795 & 0 & 21 & 15.7 \\
\hline 1796 & 0 & 16 & 13.7 \\
\hline 1797 & 0 & 6 & 7.7 \\
\hline 1798 & 0 & 4 & 4.7 \\
\hline 1799 & 1 & 7 & 5.6 \\
\hline 1800 & 0 & 14 & 11.0 \\
\hline 1801 & 0 & 34 & 51.1 \\
\hline 1802 & 1 & 45 & 35.3 \\
\hline 1803 & 3 & 43 & 18.5 \\
\hline 1804 & 1 & 48 & 21.6 \\
\hline 1805 & 2 & 42 & 25.6 \\
\hline 1806 & 1 & 28 & 13.3 \\
\hline 1807 & 0 & 10 & 5.0 \\
\hline 1808 & 0 & 8 & 3.5 \\
\hline 1809 & 0 & 3 & 1.2 \\
\hline 1810 & 0 & 0 & 0.0 \\
\hline 1811 & 0 & 1 & 0.3 \\
\hline 1812 & 0 & 5 & 4.0 \\
\hline 1813 & 0 & 12 & 9.1 \\
\hline 1814 & 3 & 14 & 10.4 \\
\hline 1815 & 0 & 35 & 16.8 \\
\hline 1816 & 1 & 46 & 30.8 \\
\hline 1817 & 7 & 30 & 28.0 \\
\hline 1818 & 5 & 30 & 21.7 \\
\hline 1819 & 0 & 24 & 19.2 \\
\hline 1820 & 20 & 16 & 10.7 \\
\hline 1821 & 24 & 7 & 4.3 \\
\hline 1822 & 6 & 4 & 3.0 \\
\hline 1823 & 0 & 2 & 1.2 \\
\hline 1824 & 3 & 9 & 5.1 \\
\hline 1825 & 6 & 17 & 14.4 \\
\hline 1826 & 6 & 36 & 28.6 \\
\hline 1827 & 18 & 50 & 44.4 \\
\hline 1828 & 1 & 64 & 57.0 \\
\hline 1829 & 8 & 67 & 59.2 \\
\hline
\end{tabular}

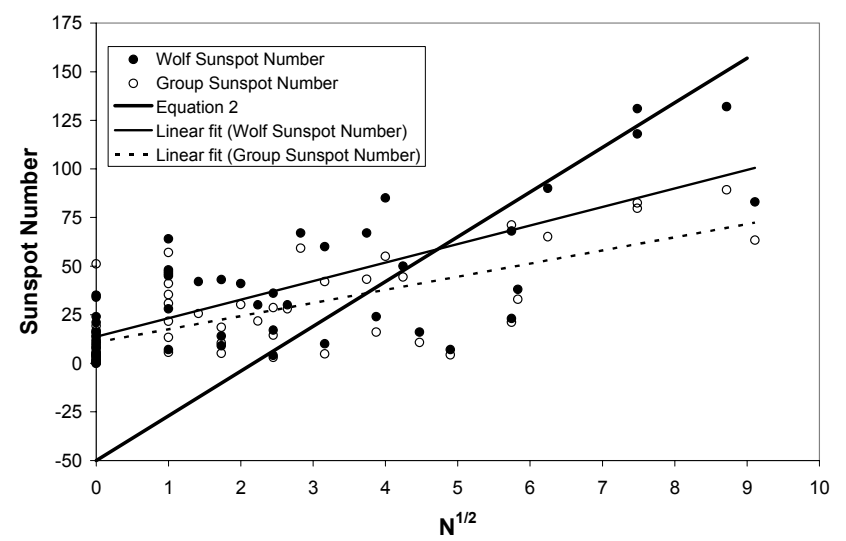

Fig. 1. Relationships between the sunspot numbers and the square root of $N$. Equation (2) is also shown.

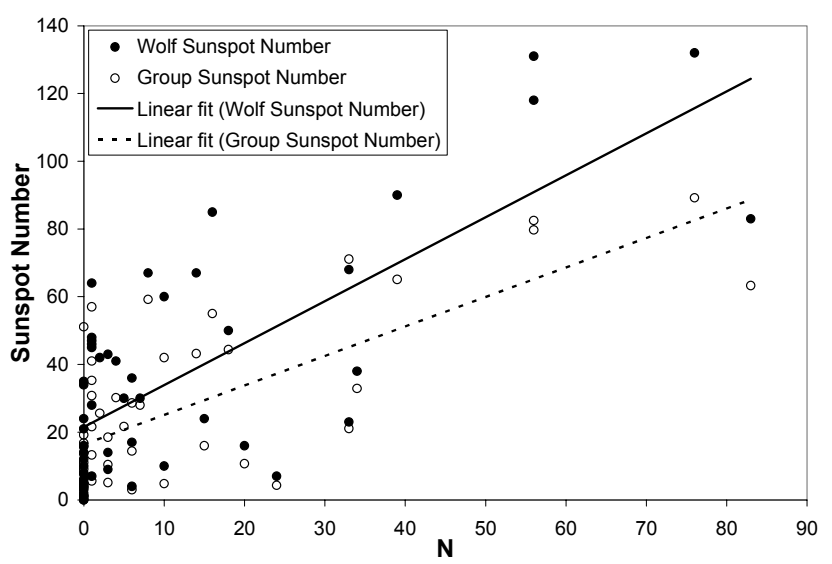

Fig. 2. Relationships between the sunspot number and $N$.

Recently, Hoyt and Schatten (1998) made a new reconstruction of solar activity from sunspot observations. This time series is known as the Group Sunspot Number, $R_{G}$, because it uses the observed number of sunspot groups. The Group Sunspot Number is designed to be more internally self-consistent (i.e. less dependent upon seeing the tiniest spots) and less noisy than the Wolf Sunspot Number. Hoyt and Schatten defined the Group Sunspot Number, $R_{G}$, as

$R_{G}=\frac{12.08}{N_{O}} \sum k_{i} G_{i}$,

where $G_{i}$ is the number of sunspot groups recorded by the $i$-th observer, $k_{i}$ is the $i$-th observer's correction factor, $N_{o}$ the number of observers used to form the daily value, and 12.08 is a normalization factor chosen to make the multiple values of the mean of the $R_{G}$ 's identical with the multiple values mean of $R_{Z}$ for 1874 to 1976 . Daily, monthly, and yearly means were derived from 1610 to the present. They calculated daily values of solar activity on 111358 days for 1610-1995, compared to 66168 days for the International Sunspot Numbers, tabulating estimates of their random and systematic errors. An important conclusion is that solar activity before 1882 is lower than that generally assumed. 


\section{Auroras and sunspot numbers during 1780-1829}

On the basis of the data presented in Table 1, the relationships between the mean annual numbers of visually observed auroras $N$ and sunspot numbers $\left(R_{Z}\right.$ and $\left.R_{G}\right)$ can be found. Figure 1 shows the relationships between the sunspot numbers and the square root of $N$. The linear fits of these data are:

$R_{Z}=(10 \pm 1) \sqrt{N}+(14 \pm 4) r^{2}=0.529$

$R_{G}=(7 \pm 1) \sqrt{N}+(11 \pm 3) r^{2}=0.500$,

where $r^{2}$ is the coefficient of determination. Figure 1 shows Eq. (2) provided by Schröder et al. (2004). Equation (2) does no seem not to be correct because if the mean annual visual observations of the auroras at middle latitudes of Europe were zero, then the Wolf number should be -50 . Moreover, even at first sight Eq. (5) seems to be a better fit to the data than Eq. (2).

Figure 2 shows the relationships between the sunspot numbers and $N$. The linear fits of these data are:

$$
\begin{aligned}
& R_{Z}=(1.2 \pm 0.2) N+(21 \pm 4) \quad r^{2}=0.541 \\
& R_{G}=(0.9 \pm 0.1) N+(17 \pm 3) \quad r^{2}=0.504 .
\end{aligned}
$$

One can appreciate that the last two values of coefficient $r^{2}$ are slightly higher than the first two. One can verify that the use of different sunspot numbers could vary the results of Schröder et al. (2004), who only use the Wolf number.

\section{Final Comments}

The estimations of brightness of expected auroras in the atomic oxygen 630-nm emission obtained by Schröder et al. (2004) could be overestimated because they used the Wolf sunspot numbers that overestimate the solar activity before 1882 (Hoyt and Schatten, 1998). Moreover, Eq. (2) of Schröder et al. (2004) modifies the true relationships between mean annual number of visual observations of the auroras $N$ at middle latitudes of Europe and mean annual Wolf number $R_{Z}$ during 1780-1829.
Acknowledgements. The author and the Topical Editor M. Lester thank D. M. Willis for his help in evaluating this paper.

\section{References}

Hoyt, D. V. and Schatten, K. H.: Group sunspot numbers: a new solar activity reconstruction, Solar Physics, 179, 189-219, reprinted with figures in Solar Physics, 181, 491-512, 1998.

McKinnon, J. A.: Sunspot Numbers, National Geophysical Data Center, NOAA, Boulder, CO, 1610-1985,1987.

Schröder, W., Shefov, N. N., and Treder, H.-J.: Estimation of past solar and upper atmosphere conditions from historical and modern auroral observations, Ann. Geophys., 22, 2273-2276, 2004, SRef-ID: 1432-0576/ag/2004-22-2273.

Truttse, Yu. L.: Upper atmosphere during geomagnetic disturbances, I, Some regular features of low-latitude auroral emissions, Planetary and Space Science, 16(2), 981-992, 1968a.

Truttse, Yu. L.: Upper atmosphere during geomagnetic disturbances, II, Geomagnetic storms oxygen emission at $6300 \AA$ and heating of the upper atmosphere, Planetary and Space Science, 16(10), 1201-1208, 1968b.

Truttse, Yu. L.: Upper atmosphere during geomagnetic disturbances, III, Some regularities in density variations, Planetary and Space Science, 17(2), 181-187, 1969.

Vitinsky, Yu. I., Kopecky, M., and Kuklin, G. V.: Statistics of the sunspot activity of the Sun, Moscow, Nauka, 65, 1986.

Waldmeier, M.: The sunspot activity in the years 1610-1960, Zurich Schulthess and Company AG, 1947. 